\title{
Politiques de logement dans \\ LES PROVINCES CANADIENNES : \\ FORCES ET FAIBLESSES
}

Housing policies in Canada's provinces: strengths and weaknesses

\author{
Maroine Bendaoud \\ Université du Québec à Montréal \\ maroineb@hotmail.com \\ https://orcid.org/0000-0003-0382-9896
}

RÉSUMÉ : Depuis les décennies d'après-guerre, les provinces canadiennes interviennent dans le secteur du logement pour soutenir les ménages dans le besoin. Cependant, très peu d'études indépendantes ont tenté de faire une évaluation globale des programmes mis en œuvre depuis. Ce texte offre une telle analyse, en observant les programmes de logement public (HLM), logement communautaire détenu par les groupes du tiers secteur et les programmes axés sur le marché locatif privé (allocation-logement et supplément au loyer). Nous nous attardons essentiellement à l'efficacité des programmes au regard de la satisfaction des besoins de logement des ménages vulnérables.

Mots-CLÉs: Politiques du logement, Canada, logement, programmes de logement, logement public, logement communautaire.

RESUMEN: Desde las décadas de la posguerra, las provincias canadienses intervienen en el sector de la vivienda para apoyar a los hogares que lo necesitan. No obstante, son pocos los estudios independientes que han intentado evaluar globalmente los programas desplegados desde entonces. Este texto ofrece un análisis en este sentido mediante la observación de los programas de vivienda pública (HML), vivienda comunitaria de los grupos del tercer sector y los programas basados en el mercado privado del alquiler (asignaciones de vivienda y suplementos al alquiler). Nos centramos especialmente en la eficacia de los programas según la satisfacción de las necesidades habitacionales de los hogares vulnerables.

Palabras clave: políticas de vivienda, Canadá, vivienda, programas de alquiler, alquiler público, vivienda comunitaria. 
RESUM: Des de les dècades de la postguerra, les províncies canadenques intervenen al sector de l'habitatge per a donar suport a les famílies amb aquesta necessitat. Tanmateix, pocs estudis independents han intentat fer una avaluació global dels programes aplicats d'aleshores ençà. Aquest text ofereix aquesta anàlisi i analitza els programes d'habitatge públic (HLM), habitatge comunitari dels grups del tercer sector i els programes basats al mercat de lloguer privat (assignació d'habitatge i suplements al lloguer). Ens centrem especialment a l'eficàcia dels programes en funció de la satisfacció de les necessitats d'habitatge de les famílies vulnerables.

Paraules Clau: polítiques d'habitatge, el Canadà, habitatge, programes de lloguer, lloguer públic, habitatge comunitari.

ABSTRACT: Since the post-war decades, Canadian provinces have intervened in the housing sector to support households in need. However, very few independent studies have attempted to make a general evaluation of the programs implemented since then. This text offers such an analysis, looking at public housing programs (PVP), community housing owned by third sector groups and programs focused on the private rental market (housing subsidy and rental supplement). Our primary focus is on the effectiveness of programs to meet the housing needs of vulnerable family units.

KEY WORDs: housing policies, Canada, housing, housing programs, public housing, community housing. 
Patrick Lagacé : Est-ce que c'est juste moi ou le principe du droit au logement, on s'en fout complètement collectivement?

François Saillant : Malheureusement, c'est le cas présentement. On ne peut pas dire que c'est une grande préoccupation. Évidemment, il arrive certains évènements qui font réagir. Par exemple, un cas d'insalubrité ces jours-ci sur le Plateau Mont-Royal qui fait jaser. Des fois il y a des pénuries de logements qui font jaser. Mais de manière générale, je ne peux pas dire que c'est une grande

préoccupation. Et le fait que ce ne soit pas une grande préoccupation de la population, pas une grande préoccupation des médias et bien ça ne devient pas non plus une grande préoccupation pour les politiciens. On n'en a pas entendu parler beaucoup durant la campagne électorale. ${ }^{1}$

$\mathrm{L}$ a citation placée en épigraphe de François Saillant, porte-parole du Front d'action populaire en réaménagement urbain (FRAPRU) pendant une trentaine d'années, dresse le portrait général. Évoqué dans la presse ou par les groupes de pression, le droit au logement soulève bien des questions et c'est pourquoi il fait l'objet de ce numéro thématique. Bien que la Déclaration universelle des droits de l'homme et le Pacte international relatif aux droits économiques, sociaux et culturels définissent l'accès au logement comme un droit pour tous les êtres humains, le cadre juridique dans les provinces canadiennes est établi par les législations internes ${ }^{2}$. Ainsi, dans un contexte où le marché privé ne réussit pas à satisfaire ce besoin humain fondamental pour tous et toutes, la réalisation du droit au logement est largement laissée entre les mains des dirigeants politiques, maîtres de l'allocation des ressources. Autrement dit, la réalisation de ce droit dépend de la volonté politique et des budgets consentis à l'aide au logement, mais pas des tribunaux. Par contre,

1. Extrait de l'émission télévisée Deux hommes en or, diffusée sur les ondes de Télé-Québec le 19 octobre 2018. En ligne, http://deuxhommesenor.telequebec.tv/emissions/141/mario-pelchat-emilie-perreault-mehdi-bousaidan-francois-saillant/41443/ francois-saillant

2. Par exemple, le Code civil du Québec pour les relations locataires-locateurs incluant les évictions, reprises, etc. et la Charte des droits et libertés de la personne pour contrer la discrimination. 
comme la citation en épigraphe l'indique, le logement abordable n'est pas une priorité gouvernementale ne recevant qu'autour de $1 \%$ du budget des provinces, sauf en de rares exceptions. En comparaison, plus de la moitié des budgets provinciaux sont consacrés à la santé et l'éducation ${ }^{3}$, des enjeux qui touchent les classes moyennes et supérieures.

Cela dit, les provinces ont tout de même mis en œuvre diverses politiques favorisant l'accès à un logement abordable, adéquat et sécuritaire. Ce texte propose d'en faire une évaluation sommaire. Bien que peu nombreuses et remontant à plusieurs années, il existe des publications qui couvrent les interventions provinciales de logement (ex. Dansereau 2005 ; Ekos Research Associates 1997 ; Fallis et al. 1995), ainsi que d'autres abordant surtout l'approche fédérale (ex. Suttor 2016, Hulchanski et Shapcott 2004 ; Sewell 1994). Une publication récente compare la production de logements sociaux au Québec et en Colombie-Britannique, en traitant des formes de subvention, ententes avec différents partenaires dont les groupes du tiers secteur, etc. (Pomeroy et al. 2019). Toutefois, le présent texte se démarque en proposant un examen critique des forces et des faiblesses des instruments de politique de logement dans les plus grandes provinces : Québec, Ontario, Alberta et Colombie-Britannique. D'abord, le logement public, connu sous l'appellation d'habitation à loyer modique (HLM). Ensuite, les logements associés au tiers secteur, à savoir les logements possédés par les organismes sans but lucratif (OSBL) ou coopératives d'habitation. Enfin, les instruments axés sur le marché locatif privé à savoir les allocations-logement et les suppléments au loyer. Chacune des sections termine par une synthèse des forces et faiblesses de chaque instrument, dans une optique de réponse aux besoins des ménages vulnérables selon le principe fondateur du droit au logement (Haut-Commissariat des Nations Unies aux droits de l'homme 2010).

3. Statistique Canada, Tableau 10-10-0024-01 (anciennement CANSIM 385-0040). 
Tableau 1. Logements ou ménages subventionnés par les provinces du Québec (QC), de l'Ontario (ON), l'Alberta (AB) et la Colombie-Britannique (CB) ${ }^{4}$

\begin{tabular}{|c|c|c|c|c|c|c|c|c|c|c|c|c|}
\hline & \multicolumn{4}{|c|}{ Secteur public } & \multicolumn{4}{|c|}{ Tiers secteur } & \multicolumn{4}{|c|}{ Secteur privé } \\
\hline & $Q C$ & $O N$ & $A B$ & $C B$ & $Q C$ & $O N$ & $A B$ & $C B$ & $Q C$ & $O N$ & $A B$ & $C B$ \\
\hline 1975 & 14466 & 85368 & 11193 & 6845 & 0 & 2145 & 0 & 5026 & 0 & 3136 & 0 & 0 \\
\hline 1995 & 64551 & 99770 & 36958 & 8032 & 14879 & 111368 & 1386 & 17023 & 62881 & 16700 & 2673 & 13338 \\
\hline 2015 & 71317 & 103670 & 42930 & $11112^{5}$ & 38800 & 116788 & 11578 & $57335^{6}$ & 103304 & & 11035 & 32732 \\
\hline
\end{tabular}

\section{Logement public}

Les logements publics communément appelés HLM sont détenus et gérés par les pouvoirs publics, au niveau provincial ou municipal, bien qu'ils aient été largement financés par le gouvernement fédéral à travers la Société canadienne d'hypothèques et de logement (SCHL). Dans les décennies qui suivirent la Seconde Guerre mondiale, certains complexes d'habitation étaient de grande taille en matière de volume, nombre d'unités, etc. (SCHL 2011, 142-6). Alors qu'au début une certaine mixité existait, les projets HLM construits ont rapidement été divisés en deux groupes : familles et aînés. La contribution des locataires est fixée à 25 ou $30 \%$ de leur revenu, avant impôt, assurant l'abordabilité des loyers. Comme la demande de logements publics a pratiquement toujours dépassé l'offre, faute de financement suffisant, les organisations gouvernementales maintiennent une liste d'attente avec un système pour classer les demandeurs. Lorsqu'un logement se libère, le demandeur

4. Les unités sont comptées de manière cumulative, incluant tous les logements ou ménages soutenus par les gouvernements provinciaux avec ou sans l'aide du gouvernement fédéral via la SCHL. Les unités du secteur privé comprennent uniquement les programmes d'allocation-logement et de suppléments de loyer, mais il n'existait pas de données agrégées pour l'Ontario en 2015. Les propriétaires (près de 7000) participants au programme québécois Allocation-logement ont été retirés. Outre les rapports annuels des agences provinciales, la plupart des données pour l'année 2015 ont été fournies par des fonctionnaires provinciaux, sauf pour l'Ontario où il s'agit de données estimées selon les chiffres du ministère du Logement et tels que reproduits dans ONPHA et CHF $(2013,26)$.

5. Concernant l'augmentation d'unités du secteur public entre 1995 et 2015 en Colombie-Britannique, ces unités appartiennent au gouvernement mais sont généralement gérées par des OSBL.

6. Ce nombre comprend environ 15000 unités de logement à but non lucratif financées uniquement par le gouvernement fédéral et transférées au gouvernement de la Colombie-Britannique en 2006. 
le plus nécessiteux au haut de la liste se voit offert l'unité qui convient à son ménage. Les ménages décident s'ils acceptent ou non, mais leur nombre de rejets est limité de même que leur choix de complexes HLM dans une zone territoriale désignée. De plus, très peu de logements sont vacants dans la mesure où les résidents qui vivent en HLM y demeurent plus longtemps à comparer aux logements du secteur privé. Malheureusement, un ménage qui s'inscrit sur une liste d'attente peut y rester de nombreuses années avant de se voir proposer un logement (Sewell 1994 ; Bendaoud 2019a).

L'Ontario est la province où la croissance de logements publics a été la plus rapide. Du milieu des années 1960 au milieu des années 1970, l’Ontario était le leader au pays (Rose 1980, 96). Par contre, cette croissance fut ralentie par l'opposition des municipalités et leurs citoyens contre les projets HLM pour familles. La production s'est poursuivie jusque dans les années 1980, à un rythme plus lent, et se concentrait essentiellement sur les projets HLM pour aînés (OHC 1984, 40). Dans les années 1960, le système de classement donnait la priorité aux familles visées par l'élimination des taudis. Dans les années 1970 et 1980, le revenu puis progressivement d'autres facteurs sociaux et liés à la santé ont été considérés (OHC 1984, 34). Des groupes particuliers se sont vu accorder la priorité dans les critères de sélection : personnes handicapées (1978), personnes souffrant de troubles mentaux ou de développement (1981), troubles psychiatriques (1986), parents mineurs (1986), femmes victimes de violence (1986), résidents permanents (1988), demandeurs d'asile (1991). De sorte que les logements publics ont de plus en plus desservi des citoyens très vulnérables, contribuant à la précarisation du profil des locataires (Smith 1995).

Sous la gouverne du premier ministre conservateur Mike Harris, la responsabilité du logement abordable, incluant celle du logement public, a été décentralisée à l'échelon municipal au tournant des années 2000. Depuis, les municipalités ontariennes ont augmenté leur stock de logements " publics » sauf que cela s'est fait d'une autre manière, soit à travers les OSBL municipaux discutés plus bas (ONPHA et CHF 2013, 26). Et bien qu'ils soient plus abordables que les loyers médians, ces nouveaux logements ne sont pas offerts à un loyer proportionné au revenu du ménage (ex. à 30\% du revenu). Ce qui retient l'attention est surtout les projets de revitalisation à Toronto, 
notamment celui de Regent Park qui est le plus ancien et le plus large complexe HLM au Canada, avec plus de 2000 unités. Le projet, qui n'est pas entièrement complété, vise à corriger plusieurs faiblesses identifiées dans les propriétés de la Toronto Community Housing Corporation (TCHC) en général :

[M] any of TCHC's 110,000 tenants are dissatisfied with the state of repair and cleanliness of their buildings. Many do not feel safe in their homes because of social disorder and criminal activity in their neighbourhoods. Vulnerable residents lack ready access to services to deal with mental health, addiction, hoarding and aging. At the same time, TCHC struggles with an ever growing backlog of capital repairs, inadequate operating funding, and the need to manage tenant issues for which the company is not adequately resourced. (Ville de Toronto 2016, 4)

La trajectoire du logement public au Québec et en Alberta est assez similaire à celle décrite en Ontario, du moins jusqu'aux années 1990 et avant la décentralisation. Au Québec, le premier projet d'habitation Jeanne-Mance construit à la fin des années 1950, situé au centre-ville de Montréal, compte près de 800 logements, mais demeure moins imposant que celui de Regent Park. Les tours d'habitation qui étaient la tendance dans les premiers projets HLM de la Société d'habitation du Québec dans les années 1960 sont progressivement abandonnées « au profit de bâtisses de deux ou trois étages, aux dimensions plus humaines » (SHQ 1992, 18). Comme en Ontario, le Québec a également connu une précarisation du profil des locataires de HLM (Baillergeau et Morin 2008). L'arrêt du financement fédéral pour les nouvelles unités de logement social en 1994, incluant de logement public, a marqué un hiatus important dans la production de logements publics au Québec et en Alberta. Cela dit, lorsque des subventions fédérales pour réaliser de nouveaux logements abordables sont redevenues disponibles au début des années 2000, le Québec et surtout l'Alberta ont connu une croissance du stock de logements de propriété publique (Bendaoud 2018). Tel que mentionné pour le cas ontarien, les nouveaux logements ne sont pas proportionnés au revenu des locataires en Alberta et ne le sont que dans une certaine proportion au Québec où une partie des ménages (moins de 50\%) bénéficie d'un supplément au loyer. 
Pour finir sur le cas de la Colombie-Britannique, l'histoire du logement public est assez brève. Elle termine en 1975 à la perte du pouvoir par le parti néodémocrate. Cela dit, le retour du même parti progressiste de 1991 à 2001 n'a pas non plus ramené le logement public au centre de l'élaboration de la politique de logement, résolument tournée vers d'autres instruments (Bendaoud 2018). De plus, dans les dernières années, le titre de propriété d'une partie des HLM provinciaux a été transféré à des OSBL choisis pour leur forte expérience de gestion.

La formule du logement public HLM permet-elle de satisfaire les besoins de logement des ménages vulnérables ? Disons que ses forces sont surtout d'offrir de logements de différentes tailles (incluant pour les familles nombreuses) aux plus démunis de la société, à un coût toujours abordable puisqu'il est calculé selon un pourcentage du revenu du ménage. Les HLM ont aussi permis de créer de nouveaux logements à une époque où des taudis étaient encore assez présents dans certains marchés privés. Cependant, la formule a aussi plusieurs faiblesses. Vu les restrictions budgétaires et l'objectif de maximisation du nombre d'appartements au moment de la construction, en plus d'un grave manque de fonds pour l'entretien et les rénovations, la qualité des bâtiments a souvent été déplorée. Si l'on ajoute à cela la précarisation progressive de la clientèle, force est de constater que les HLM n'ont pas toujours créé des milieux de vie enviables (voir Bendaoud 2019a). À dire vrai, ils ont souvent été considérés comme des logements de dernier recours (Sousa 2013). Ces critiques sont surtout dirigées vers les plus vieux projets HLM, qui contiennent beaucoup d'unités et logent les familles (non les aînés). Par contre, il faut souligner les efforts d'organismes publics gestionnaires des HLM, souvent aidés par les ordres de gouvernement, qui visent à améliorer la qualité de vie dans les projets HLM et ce d'une multitude de façons.

\section{OSBL et coopératives d'habitation}

L'ensemble de logements d'un OSBL est possédé et géré par l'OSBL lui-même, en tant que personne morale, comptant certains employés ou bénévoles. Les OSBL sont souvent fondés par des groupes de la société civile, par exemple les groupes communautaires, culturels ou confessionnels, sociétés de bienfaisance, etc. Les coopératives appartiennent collectivement à leurs 
membres/occupants qui s'impliquent beaucoup dans leur gestion, sans toutefois pouvoir en tirer un gain pécuniaire en cas de vente des actifs. Caractérisés par de petits ensembles de logements et un esprit de " communauté », les OSBL et coopératives d'habitation ont connu un essor important dans les années 1970 comme nous le verrons plus bas (SCHL 2011, 143-7 ; Bouchard et al. 2010, 93-5 ; Sousa 2013). Bien qu'ils ne fonctionnent pas de la même manière en ce qui concerne le calcul du loyer et la sélection des locataires, la plupart des OSBL et coopératives d'habitation font aussi face à un manque de logements disponibles dans leurs bâtiments, ce qui génère également des listes d'attente. Les locataires actuels y demeurent aussi plus longtemps, vu le prix des loyers en dessous de ceux sur le marché privé. Les OSBL et les coopératives d'habitation sont souvent groupés sous le terme de « tiers secteur».

La Colombie-Britannique se démarque par la montée précoce des OSBL d'habitation, avant même l'arrivée des programmes fédéraux des années 1970. En effet, des milliers d'unités ont été développées pour les aînés grâce à une loi provinciale datant de la fin des années 1950 (Elderly Citizens' Housing Aid Act). Les OSBL logeaient les aînés autonomes pour la plupart, mais certains avaient des besoins plus particuliers. Le ministère responsable encourageait les groupes à but non lucratif tels que les clubs philanthropiques, églises de différents acabits et groupes ethniques (par exemple d'Europe de l'Est) à construire différents types de logements pour personnes âgées. Dans les années 1970, ce sont surtout les coopératives qui construisaient des logements destinés aux familles. En matière de profil socio-économique, les complexes HLM avaient la réputation de concentrer les ménages pauvres. Les projets d'habitation développés par les OSBL et les coopératives contenaient quant à eux un mélange d'unités non subventionnées, où les locataires payaient un loyer relativement bas (low end of market), et d'unités louées par des locataires payant un loyer proportionné à leur revenu. Les projets d'habitation du tiers secteur étaient plus petits que les complexes HLM et conçus pour s'insérer dans le quartier ou la trame urbaine (Colombie-Britannique, Department of Housing 1975a).

De manière générale, la montée du tiers secteur en habitation dans les années 1970 est associée à la mixité sociale (Dennis et Fish 1972 ; Fallis et al. 1995). C'était le cas en Colombie-Britannique, particulièrement pour les 
coopératives. Celles-ci pouvaient loger des ménages au revenu moyen considérant qu'il n'y avait pas de restriction sur le revenu. En fait, la contrainte était placée sur la moyenne des revenus des membres de la coopérative, cette moyenne devant être en dessous de la limite fixée par la SCHL (Colombie-Britannique, Department of Housing 1975b, 10-11). Par contre, les choses ont changé dans le milieu des années 1980 comme l'indique cette publication qui réorienta la politique de logement britanno-colombienne :

Despite these large expenditures, households other than the most needy are being assisted. The existing program [anciennement l'article 56.1 de la Loi nationale sur l'habitation, axé sur le tiers secteur] guidelines require only that $15 \%$ of households in each development be most in need; the remainder can be moderate or middle income. Consequently, only about $1.5-2.0 \%$ of all households most in need nationally are being housed each year through the current social housing program. A survey of all co-operatives in Vancouver indicated that $40 \%$ - $45 \%$ of residents could afford non-subsidized rental housing, and that only about half of the residents in housing co-operatives surveyed were high priorities for assistance. Many households receiving social housing benefits do not require them and more people in need could be assisted with the dollars available. (BC Housing 1986, 1-2).

L'année 1986 marqua une réflexion de la part des élites politico-administratives et même des acteurs de la société civile (Cosh et al. 1986) dans l'attribution des logements sociaux (nouveaux et certains déjà construits) en Colombie-Britannique, afin d'en resserrer les critères et cibler les ménages plus nécessiteux. Cette réorientation a perduré une trentaine d'années, soit au moins jusqu'au plan lancé par le gouvernement néodémocrate de John Horgan en 2018 pour surmonter le problème de la cherté des logis dans l'ensemble de la province (Colombie-Britannique 2018). Pour en revenir aux groupes du tiers secteur, il est clair que les OSBL d'habitation sont le principal véhicule pour la réalisation de logements abordables de la province, depuis le milieu des années 1970. Outre le fait que les logements produits par les OSBL sont moins coûteux par unité que ne le sont les logements publics, l'expertise de ces organisations en ce qui a trait à la gestion des propriétés et l'offre de services spécialisés et adaptés aux clientèles qu'ils desservent est aussi un facteur clé dans l'adoption du modèle sans but lucratif (Bendaoud 2018). Cela dit, même le plan ambitieux du gouvernement néodémocrate, si 
audacieux et lourd d'engagements soit-il, ne parviendra pas à répondre à tous les ménages dans le besoin, ces derniers étant simplement trop nombreux.

Au Québec, les groupes du tiers secteur sont moins dominants dans le paysage du logement social qu'en Colombie-Britannique (tableau 1, plus haut), vu la place des autres acteurs, mais ils ont également connu une forte ascension depuis la fin des années 1970. Le Québec compte aussi certains gros joueurs, détenteurs de centaines unités de logement, qui ont développé des expertises particulières auprès de diverses clientèles. Cela dit, une des spécificités du Québec est probablement la montée des groupes de ressources techniques, les GRT, qui sont devenus le moteur de presque tous les projets d'habitation du tiers secteur depuis le tournant des années 1980. Ils étaient la cheville ouvrière du démarrage des projets et sont devenus aujourd'hui des entreprises prospères d'économie sociale ${ }^{7}$. Tout cela étant dit, la clientèle logée par les groupes du tiers secteur est aussi discutée depuis fort longtemps. $\mathrm{Au}$ milieu des années 1980, comme cela se produisait simultanément en Colombie-Britannique, la latitude des programmes était remise en question : «Actuellement, on exerce peu de contrôle sur les clientèles. Pourtant l'importance de la subvention [fédérale et provinciale] justifierait l'idée d'une vérification plus systématique de la clientèle bénéficiaire de ces programmes »(Québec 1984, 69). La principale recommandation de la vaste étude sur les programmes québécois de logement soutenait que ceux-ci doivent : « rechercher l'équité horizontale (à revenu égal aide égale pour tous) et l'équité verticale (aide inversement proportionnelle au niveau de revenu) à l'égard des bénéficiaires dans les interventions de nature financière » (Québec 1984, 95-6).

Depuis 1997, le principal programme de réalisation de logements abordables québécois, AccèsLogis, prédétermine le pourcentage minimal de ménages à faible revenu qui doit être logé dans chaque nouveau projet. Cela dit, cela n'enraye pas un problème persistant dans la sélection des locataires : le biais en faveur des « bons locataires ». En effet, vu la rareté des logements abordables et les listes d'attente, certaines coopératives et certains OSBL peuvent se permettre d'être très sélectifs dans le choix des candidats,

7. À Montréal, le GRT Bâtir son quartier est un des plus connus. 
particulièrement si les logements sont situés dans quartiers prisés. Cela se produit dans les nouvelles constructions (Desage 2017), mais aussi dans les plus anciennes où, au fil du temps, des administrateurs ont abandonné la mission sociale de l'organisme et ont décidé de ne plus loger ou offrir une aide substantielle aux ménages vulnérables (voir CQCH 2007 et Bendaoud $2019 b, 5,8)$. Leur argument se résume à ceci : le soutien aux plus démunis est la responsabilité du gouvernement, pas la leur. Une étude empirique récente réalisée à Montréal notait d'ailleurs que « l'écart entre le revenu des ménages habitant en coopérative et celui des copropriétaires de logements abordables est parfois mince » (Germain et al. 2017, 3)

L'Ontario a aussi connu une poussée du développement de logements possédés par les groupes du tiers secteur dès les années 1970. Cela s'est fait en grande partie en réaction aux HLM publics. Tel que mentionné plus haut, la province a radicalement réorienté sa politique de logement à partir de cette période. Fait intéressant, alors que les autres provinces concentraient leur budget en habitation sur les plus démunis à partir du milieu des années 1980, selon l'entente convenue avec Ottawa mais largement endossée par les provinces, l'Ontario a fait cavalier seul sur le maintien de la mixité. En fait, la province a utilisé les fonds fédéraux, puis ajouté les siens, pour les unités destinées aux plus démunis. Cependant, au sein des mêmes projets d'habitation, la province finançait unilatéralement des appartements pour les ménages à revenu modéré afin de conserver la mixité et l'acceptabilité par les communautés locales (Wilson 1989, 22). Depuis la décentralisation municipale au début des années 2000 et la reprise plutôt timide du développement de nouveaux logements abordables, le portrait ressemble à celui du Québec où une certaine mixité existe mais où les personnes vulnérables (itinérants, aînés en perte d'autonomie, femmes victimes de violence, etc.) reçoivent une attention prioritaire dans le financement. Les OSBL sont également le principal véhicule de développement de nouveaux logements abordables, mais notons que les OSBL municipaux sont aussi des joueurs importants. Ces OSBL ont été créés à partir des années 1970 et certains sont apparus plus tard (par exemple la SHDM à Montréal). À l'origine ils étaient bien distincts des organismes gérant les HLM. Sauf que depuis la décentralisation municipale en Ontario, ces organisations ont commencé à porter les deux casquettes gérant leurs propres unités ainsi que celles héritées des anciens programmes 
de logement public. En Ontario, ces OSBL ont aussi développé une part importante des nouveaux logements grâce aux opportunités de financement rendues disponibles par les ordres supérieurs de gouvernement (ONPHA et CHF 2013, 26).

Enfin, l'Alberta est la province des quatre où les groupes du tiers secteur ont historiquement eu une place beaucoup plus faible. Toutefois, depuis le retour des nouveaux investissements provinciaux dans le logement au début des années 2000, toujours sous la gouverne de Ralph Klein qui avait fermé le robinet pendant son premier et deuxième mandats, les groupes du tiers secteur ont développé un peu plus de nouveaux logements abordables que le secteur privé et public. Cela n'était toutefois pas le fruit d'une politique habilement orchestrée ou même d'une décision réfléchie, mais plutôt le résultat des processus d'appels d'offres lancés aux organismes désireux de fournir des logements à travers le même type d'opportunités de financement discutées plus haut (Bendaoud 2018).

Quel bilan dresser des groupes du tiers secteur en ce qui a trait à leurs réponses aux besoins des démunis ? Comme pour le logement public, disons que le logement communautaire a aussi ses forces et ses faiblesses. Parmi ses forces nous pouvons très certainement compter le développement de milieux de vie plus désirables (en matière de site et de clientèle) pour les résidents, si on les compare aux logements publics et ce qu'ils sont devenus. Le délai de construction est toutefois similaire : dans les deux cas il faut compter au moins deux à trois ans. Certains OSBL ont perfectionné leur approche dans la gestion de propriété et l'offre de services adaptés aux besoins et situations particulières de leurs clientèles : femmes victimes de violence, aînés en perte d'autonomie, personnes avec des problèmes de santé, ex-itinérants, etc. Des OSBL et surtout des coopératives adoptent également des pratiques très démocratiques à travers la gestion collective (Bouchard et al. 2010). Par contre, ce dernier élément est aussi à la source de la principale faiblesse : le biais de sélection envers les « bons locataires » faisant en sorte que nombreux ménages en situation précaire, ayant un faible capital social et niveau d'éducation, sont exclus. Notons aussi que les résidents des coopératives ou OSBL qui payent un loyer moins cher que le coût du marché - sans toutefois bénéficier d'un loyer proportionné dû à leur pauvreté - ont moins d'incitation à déménager que les personnes sur le marché privé et même celles en HLM. Si 
le ménage locataire d'un HLM voit sa situation financière s'améliorer (hausse salariale, intégration d'un(e) conjoint(e) au ménage, etc.), le loyer proportionné à 25 ou $30 \%$ du revenu atteindra un point où le ménage quittera de lui-même pour trouver mieux sur le marché privé. Il laissera ainsi sa place à un ménage défavorisé sur la liste d'attente. À l'opposé, si l'on prend des coopératives issues d'anciens programmes qui logeaient essentiellement des familles salariées et où les loyers sont plus bas que les prix du marché pour l'ensemble des résidents, le loyer ne sera pas majoré si le ménage accroît son revenu. Ce ménage a donc tout intérêt à préserver sa place dans la coopérative (voir par ex. Tison 2019).

\section{Instruments axés sur le marché privé}

Les instruments axés sur le marché locatif privé utilisent les logements déjà bâtis. Ils ont été conçus chronologiquement après ceux visant la construction de logements sociaux. Le programme de supplément au loyer dans sa version locative privée, développée par la SCHL au niveau fédéral, permet aux pouvoirs publics de convenir d'un accord avec des propriétaires privés pour louer leurs logements à des ménages pauvres qui paieront un loyer proportionné à leur revenu. Ce programme étant géré par les organismes gestionnaires des HLM et les bénéficiaires sélectionnés à partir des mêmes listes d'attente, ceux-ci payent le même coût qu'en HLM à savoir 25 à 30\% de leur revenu avant impôt (SCHL 2011, 146). Les allocations-logement sont des initiatives provinciales apparues à partir de la fin des années 1970. Ils prennent la forme de transferts en espèces (sans suivre une formule proportionnelle au revenu) généralement fournis à tous les ménages admissibles, c'est-à-dire sans liste d'attente. Le ménage peut choisir son logement sur le marché privé et si la personne déménage, la subvention déménage avec elle. C'est ce qui confère à l'instrument son caractère « portatif », attaché à la personne et non à l'unité. Cela dit, les allocations-logement peuvent également être utilisées pour des appartements détenus par des OSBL, dans les unités où le loyer est fixé au prix du marché. Notons également que l'allocation peut être appliquée pour le logement où le ménage réside avant que la subvention démarre, lui permettant ainsi de demeurer où il est. Les transferts sont confidentiels au sens où les propriétaires privés ou à but non lucratif ne savent généralement pas quels 
locataires bénéficient de cette aide financière, réduisant ainsi le risque d'augmentation de loyer (Steele 2007). Précisons que les aides peuvent atteindre plusieurs centaines de dollars par mois dans toutes les provinces de l'étude à l'exception du Québec où le maximum est de $80 \$$. Le tableau 1 présenté plus haut intègre les données pour les deux formules, supplément au loyer privé et allocations-logement, mais ce sont ces dernières qui seront davantage discutées vu leur nombre de bénéficiaires supérieur. Dans les deux cas, encore une fois, le manque de financement des programmes ne permet pas de fournir un logement abordable et accessible à tous et toutes.

Le professeur émérite en travail social Andrew Armitage était sous-ministre adjoint au ministère du Logement de Colombie-Britannique lorsqu'il a présidé l'Interprovincial Task Force on Shelter Allowances and Rent Scales. Il résume dans cet extrait-ci les avantages du programme Shelter Aid For Elderly Renters (SAFER) lancé en 1977 et qui couvrait 75\% du affordability gap, c'est-à-dire l'écart entre la somme équivalente à $30 \%$ du revenu du ménage et le coût actuel du loyer.

First, SAFER covers all low income elderly renters, hence it is universal for those in need. Second, it allows senior citizens who are able to stay in present accommodation to do so if they choose. ${ }^{8}$ Third, since it works in conjunction with private sector accommodation and has a controlling mechanism built into the formula, it required far less subsidy on a per unit basis. Fourth, SAFER greatly reduces the inequity between the treatment afforded [to] those living in the public/non-profit sector versus those living in the private market (Interprovincial Task Force on Shelter Allowances and Rent Scales 1978, 17).

Trente ans plus tard, le gouvernement de Colombie-Britannique a créé une nouvelle allocation-logement dédiée aux familles détenant un emploi, le Rental Assistance Program (RAP). D'autres formules similaires ont aussi été créées pour toucher les populations itinérantes ou à risque de le devenir (voir BC Housing 2015, 16). Ce qui ne veut pas dire qu'aucune critique ne leur ait faite. Seth Klein, alors directeur du Canadian Centre for Policy Alter-

8. Il ajoutait : « [I]t is not always desirable to force a senior citizen to move to a different neighborhood. It had been discovered that many senior citizens were moving into public/non-profit housing projects as a matter of last resort, and really would have preferred to stay in their existing accommodation if they could have afforded to do so » (Interprovincial Task Force on Shelter Allowances and Rent Scales 1978, 17). 
natives disait du RAP, l'allocation pour familles salariées, «Such programs may indeed be valuable to some families, but they do nothing to create new low-income housing stock [...] And the low take-up rate for the RAP indicates that most low-income families have not been adequately encouraged to access the program » (Klein 2013). Dans une publication précédente, Klein et Lorraine Copas du Social Planning and Research Council of BC écrivaient : " Given the importance that stable and affordable housing plays in promoting family well-being, it ought to be possible to proactively offer these grants to all families with incomes that fall below a given income level, data the government can access from income tax returns » (Klein et Copas 2010, 8). Autrement dit, des critiques se faisaient entendre sur le fait que le programme ne crée pas de nouveaux logements et qu'il y a un manque d'information ou d'incitation des familles admissibles jumelé à l'inertie du gouvernement à cet égard.

Le Québec a aussi emboîté le pas en créant Logirente en 1980. Cette allocation-logement pour aînés était quasi-identique à SAFER en Colombie-Britannique (clientèle, mécanisme et générosité de l'aide), étant toutefois un peu plus englobante en acceptant que les aînés propriétaires avec des revenus modestes y participent. Les arguments justifiant la mise en place du programme faisaient écho à ceux présentés plus haut : « Le programme permet aux personnes âgées de rester dans leur logement ou de s'en procurer un qui leur convient en allégeant leur charge financière » (Québec 1984, 69-70). Le gouvernement soulignait aussi que « L'allocation-logement est moins coûteuse que les déficits d'exploitation des HLM neufs. Avec une même somme, on peut donc rejoindre davantage de ménages dans le besoin. L'allocation logement est aussi un programme de gestion simple et peu coûteuse, ne nécessitant pas le maintien d'importants effectifs administratifs » (Québec 1984, 98).

En 1997, le gouvernement québécois a développé un nouveau programme qui fusionnait Logirente à un autre programme administré par le ministère responsable de l'aide sociale et ciblant les familles. La fusion des deux a donné le programme toujours en vigueur Allocation-logement, couvrant à la fois les aînés et les familles, en emploi ou recevant l'aide de dernier recours. L'objectif était d'harmoniser les deux programmes pour des raisons d'équité 
(Trudel 1996, 4). Par contre, cela a aussi eu un impact sur la générosité du programme. Comme SAFER en Colombie-Britannique, Logirente couvrait $75 \%$ de l'écart entre $30 \%$ du revenu du ménage et le coût de son loyer. Le programme de l'ancien ministère de la Sécurité du revenu couvrait quant à lui $50 \%$. Or le programme unifié Allocation-logement a coupé la poire en deux à $66,66 \%$. Sauf que le montant de l'aide n'a pas été indexé ce qui explique que l'aide moyenne était de 55\$ par mois en 1998 (SHQ 1999, 21) comparativement à $61 \$$ aujourd'hui, le montant maximal étant fixé à $80 \$$ depuis de nombreuses années (SHQ 2018). Autrement dit, l'aide versée n'a jamais vraiment augmenté depuis une bonne vingtaine d'années, ce qui est très problématique. Néanmoins, l'âge des aînés a progressivement été abaissé pour atteindre 50 ans aujourd'hui et ainsi élargir la portée du programme. Dans tous les cas, les groupes de défense des mal-logés comme le FRAPRU (2016) ne manqueront pas de critiquer avec véhémence la faible générosité du programme. Cela était moins vrai dans le cas de Logirente entre 1980 et 1997 , mais les choses ont évolué pour le pire dans ce cas-ci.

En Alberta, c'est après la crise du logement au milieu des années 2000 que le groupe de travail, chargé par le premier ministre de trouver des solutions, a suggéré la création d'une allocation-logement. Les membres du groupe de travail en sont venus à la conclusion que "The more timely and efficient solution to get people into affordable housing is to increase the current allocation to rent supplements [...] A portion of the RSP [rent supplement program] allocation would be used for a pilot project to test the feasibility of attaching subsidies to qualified individuals, rather than to units » (AAHTF 2007, 17). Ce projet pilote est devenu le Direct to Tenant Rent Supplement, un programme permanent d'allocation-logement qui rejoint aujourd'hui plus de ménages que le supplément au loyer privé. Contrairement aux deux autres provinces discutées, le programme est administré par les organismes publics gestionnaires des HLM qui ont un nombre limité d'allocations-logement à attribuer, créant des listes d'attente et minant ainsi l'équité (horizontale) du programme.

Enfin, dans le cas de l'Ontario, la province est la première à avoir utilisé le supplément au loyer privé au début des années 1970. Avant la décentralisation de la responsabilité du logement abordable au tournant 
des années 2000, aucune allocation-logement n'avait été mise en œuvre bien que des conversations sérieuses avaient eu lieu à cet égard. Des données exactes pour toutes les municipalités de la province ne sont pas accessibles, mais les informations disponibles suggèrent que la grande majorité des villes ont fait l'utilisation d'une forme d'allocation-logement depuis le milieu des années 2000 (Suttor 2016, 165 ; Bendaoud, à paraître).

Avec du recul, quel bilan tirer quant à l'efficacité des instruments axés sur le marché privé pour répondre aux besoins des personnes défavorisées ? Le supplément au loyer privé a l'avantage d'offrir des milieux de vie préférables en comparaison aux HLM, mais à un coût aussi abordable pour le ménage (Leloup et Morin 2012). Si l'on prend le cas des allocations-logement, au final le principe est le même que l'assistance sociale ou tout autre transfert en espèces. Il s'agit de donner immédiatement aux ménages pauvres la capacité de se payer et de choisir eux-mêmes un logement ${ }^{9}$, bien qu'ils ne peuvent pas utiliser cette allocation-logement pour un loyer déjà subventionné (ex. HLM ou coopérative). Puis, les fonds publics investis servent uniquement à soutenir les démunis, pour le temps qu'ils le sont. Dès que le ménage quitte la pauvreté, il cesse immédiatement de recevoir l'allocation-logement et ne profitera donc plus d'un loyer subventionné. En contrepartie, les allocations-logement ont évidemment plusieurs faiblesses : la faible générosité du programme québécois n'abaisse pas suffisamment la contribution du locataire au loyer, elles ne créent pas de nouveaux logements ce qui est peu utile en période de pénurie, n'affectent pas les caractéristiques de l'habitat pour les gens avec des besoins particuliers, la majorité des bénéficiaires potentiels n'appliquent en partie à cause d'un manque d'information, un tel programme peut être démantelé facilement (Bendaoud 2019a, 325). Ce à quoi nous pouvons ajouter que ces programmes n'offrent pas de sécurité d'occupation et que les familles nombreuses ou à problèmes multiples peuvent se retrouver

9. Des observateurs se demandent parfois si les allocations-logement seront captées par les propriétaires privés qui en profiteront pour imposer des augmentations de loyer abusives. D'abord, la subvention est confidentielle. Ensuite, en cas d'augmentation de loyer, le ménage aura à en payer une partie de sa poche alors il a tout à gagner à être vigilant. Enfin, le recours à l'arbitrage auprès d'instances comme la Régie du logement est envisageable, comme c'est le cas pour n'importe quelle hausse abusive. Tous ces éléments ont déjà été réfléchis il y a des dizaines d'années (voir Québec 1984, 98). 
dans une situation où il leur est quasi-impossible d'accéder à un logement convenable dans le marché privé, malgré cette allocation.

\section{Conclusion}

Dans les quatre provinces à l'étude, différentes solutions aux problèmes de logement des personnes vulnérables ont été mises en œuvre depuis l'aprèsguerre. Ayant chacune leurs forces et leurs faiblesses, une évaluation de celles-ci s'imposait tant sur le plan théorique que pratique, pour alimenter le débat intellectuel et éclairer la prise de décision. Dans le cas des logements publics HLM, ils ont permis d'offrir des habitations toujours abordables pour les ménages. Par contre, le sous-financement au moment de la construction et en matière de rénovation, en plus de la précarisation progressive du profil des locataires souvent déjà très pauvres, ont fait que la formule HLM a essuyé plusieurs critiques. Les habitations du tiers secteur appartenant aux OSBL et aux coopératives d'habitation offraient des milieux de vie plus enviables que les projets HLM, car ils favorisaient davantage la mixité et les plus petits développements. En contrepartie, la principale critique qui leur ait fait est d'être trop sélectifs dans leur choix de locataires faisant en sorte que les plus vulnérables ne parviennent pas à y habiter. Dans le cas des instruments axés sur le marché privé, le supplément au loyer abaisse le coût du loyer au niveau d'un HLM, mais en offrant des environnements physiques souvent préférés par les locataires. L'allocation-logement permet aux personnes démunies de recevoir l'aide rapidement et normalement sans liste d'attente, puis de choisir où elles veulent résider, incluant leur appartement actuel. Étant donné que la subvention n'engage pas de coûts de construction comme les autres formules, le même budget permet d'aider beaucoup plus de ménages. En revanche, ce ne sont pas toutes les personnes admissibles qui en font la demande (cela est aussi vrai pour les HLM et les logements du tiers secteur), la faible générosité du programme québécois ne permet pas de rendre le loyer réellement abordable et enfin un ménage vulnérable peut quand même éprouver beaucoup de difficulté à trouver un logement malgré la subvention. 


\section{Bibliographie}

Alberta Affordable Housing Task Force (AAHTF). 2007. Housing First An Investment with Return in Prosperity. Edmonton : Municipal Affairs and Housing.

Baillergeau, É. et P. M. 2008. « HLM et lien social ». Dans Paul Morin et Évelyne Baillergeau, dir., L'habitation comme vecteur de lien social. Québec : Presses de l'Université du Québec, 121-130.

BENDAOUd, M. 2019a. « The privatization of housing assistance: Are housing allowances eroding government accountability? ». Canadian Public Administration 62 (2) : 312-332.

. 2019b. La fin des conventions d'exploitation liant le gouvernement fédéral aux OSBL : difficultés, solutions et impacts. Montréal : Réseau québécois des OSBL d'habitation (RQOH).

2018. « Public Versus Non-profit Housing in Canadian Provinces: Learning, History and Cost-Benefit Analysis ». Dans Claire Dunlop, Claudio Radaelli et Philipp Trein, dir., Learning in Public Policy: Analysis, Modes and Outcomes. Londres : Palgrave Macmillan, 167-89.

Bouchard, M. J., W. Frohn et R. Morin. 2010. « Le logement communautaire au Québec : apports et limites d'une innovation sociale ». Lien social et Politiques (63) : 93-103.

British Columbia Housing Management Commission (BC Housing). 2015. Program Guide. Burnaby : BC Housing.

1986. A Social Housing Report For British Columbia. Burnaby : BC Housing.

Colombie-Britannique. 2018. Homes for B.C. A 30-point Plan for Housing Affordability in British Columbia. Victoria : Gouvernement de Colombie-Britannique.

Colombie-Britannique, Department of Housing. 1975a. Policy Review. Victoria : Department of Housing.

1975b. Housing For People. Programs of the British Columbia Department of Housing. Victoria : Department of Housing.

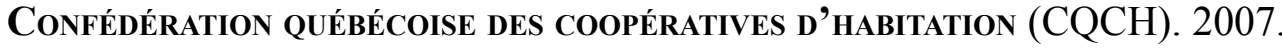
Fin des accords d'exploitation : défis et opportunités pour les coopératives d'habitation québécoises. Préparé par Allan Gaudreault. Québec : CQCH. 
Cosh, J. C., S. M. Tratch et J. Cutt. 1986. Commission of Inquiry. An Inquiry into Social Housing for British Columbia : Common Ground in Meeting Core Need. Victoria : Lieutenant Governor.

Dansereau, F., dir. 2005. Politiques et interventions en habitation : analyse des tendances récentes en Amérique du Nord et en Europe. Sainte-Foy : Presses de 1'Université Laval-SHQ.

Dennis, M. et S. A. Fish. 1972. Le logement des bas salariés: Sommaire et recommandations. Ottawa: SCHL.

Desage, F. 2017. « Les exclus de l'inclusion. Construire du logement social en temps d'austérité et de mixité (France-Québec) » Espaces et sociétés $3: 15-32$.

Ekos Research Associates. 1997. Cost-Effective Housing in British Columbia: A Comparison of Non-Profit and Market Housing. Submitted to BCHMC [BC Housing]. Ottawa : Ekos Research Associates Inc.

Fallis, G., M. Poulton, L.B. Smith, M.Y. Seelig, J. H. Seelig et J. Sewell, dir. 1995. Home Remedies : Rethinking Canadian Housing Policy. The Social Policy Challenge 6. Toronto : C.D. Howe Institute.

Front D'ACTION POPULAIRE EN RÉAMÉNAGEMENT URBAIN (FRAPRU). 2016. « Le nombre de ménages recevant de l'allocation-logement chute continuellement depuis l'an 2000. »En ligne, www.frapru. qc.ca/le-nombre-de-menages-recevant-de-lallocation-logement-chute-continuellement-depuis-lan-2000

Germain, Annick, Xavier Leloup, Damaris Rose et Juan Torres. 2017. La qualité de vie dans les projets résidentiels denses. Balises concernant la cohabitation en contexte de mixité. INRS Urbanisation, culture et société.

Haut-Commissariat des Nations Unies aux droits de l'homme. 2010. Fiche d'information no 21 : Le droit à un logement convenable. Genève : Office des Nations Unies.

Hulchanski, J. David et Michael Shapcott, dir. 2004. Finding Room. Policy Options for a Canadian Rental Housing Strategy. Toronto : Center for Urban and Community Studies/University of Toronto Press.

KLEIN, S.. 2013. « Social Housing Reality Check: Government's own numbers reveal modest investment in new social housing ». The Tyee. March 29. https://thetyee.ca/Opinion/2013/03/29/BC-Real-SocialHousing-Numbers/ 
KLeIN, S. et L.Copas. 2010. Unpacking the Housing Numbers: How much new social housing is BC building? Vancouver : CCPA et Sparc BC.

Inter-provincial Task Force on Shelter Allowances and Rent Scales FOR Senior Citizens. 1978. Report of the Inter-provincial Task Force on Shelter Allowances and Rent Scales for Senior Citizens. Toronto : Ministry of Housing.

Leloup, X. et P. Morin. 2012. Les modes d'allocation du logement social ont-ils un effet sur le bien-être et la santé des familles? Une comparaison des programmes d'habitation à loyer modique (HLM) et de supplément au loyer (PSL) à Montréal. Rapport remis à la Société canadienne d'hypothèques et de logement. Ottawa : SCHL.

Ontario Non-Profit Housing Association (ONPHA) et Co-operative Housing Federation (CHF) of Canada - Ontario Region. 2013. Where's Home 2013. Toronto: ONPHA et CHF.

Ontario Housing Corporation (OHC). 1984. Ontario Housing Corporation 1964-1984. Toronto: OHC.

Pomeroy, S., N. Gazzard et A. Gaudreault. 2019. Promising practices in affordable housing: Evolution and innovation in BC and Quebec. Canadian Housing Policy Roundtable.

QuÉBEc. 1984. Se loger au Québec : une analyse de la réalité un appel à l'imagination. Québec : Gouvernement du Québec.

Rose, A. 1980. Canadian Housing Policies 1935-1980. Toronto : Butterfield and Co.

Sewell, J. 1994. Houses and Homes: Housing for Canadians. Toronto : James Lorimer \& Co.

Sмiтh, N. 1995. "Challenges of Public Housing in the 1990s: The Case of Ontario, Canada ». Housing Policy Debate 6 (4) : 905-931.

Société CANADIENNE D'HYPOTHÈQUES ET DE LOGEMENT (SCHL). 2011. L'observateur du logement au Canada 2011. Neuvième édition d'une série annuelle. Ottawa : SCHL.

Société D'Habitation du Québec (SHQ). 2018. Rapport annuel 2017-2018. Québec : SHQ. . 1999. Rapport annuel 1998. Québec : SHQ.

1992. Une histoire en trois mouvements. Québec : SHQ.

SousA, J. 2013. Building a Co-operative Community in Public Housing: The Case of the Atkinson Housing Co-operative. Toronton : University of Toronto Press. 
STEele, M. 2007. « Canadian housing allowances ». Dans Peter A. Kemp, dir., Housing Allowances in Comparative Perspective. Bristol : Policy Press, 61-85.

Sutror, G. 2016. Still Renovating: A History of Canadian Social Housing Policy. Montreal and Kingston: McGill-Queen's University Press.

Tison, M. 2019. « Quarante années (heureuses) en coop ». La Presse, 14 avril. En ligne, https://www.lapresse.ca/affaires/2019-04-14/ vivre-en-cooperative-s-investir-plutot-qu-investir

Trudel, R. 1996. «Vers une réforme de l'aide gouvernementale en habitation ». Mémoire au Conseil des Ministres. 11 juillet.

Ville de Toronto. 2016. Transformative Change for TCHC. A Report from the Mayor's Task Force on Toronto Community Housing. Toronto: Ville de Toronto. 
